\title{
PERFIL DOS IDOSOS COM ALTERAÇÕES COGNITIVAS EM DIFERENTES CONTEXTOS DE VULNERABILIDADE SOCIAL
}

\author{
Profile of elderly people with cognitive alterations in different contexts \\ of social vulnerability \\ Perfil de los ancianos con cambios cognitivos en diferentes contextos de \\ vulnerabilidad social
}

\section{RESUMO}

Estudo de natureza quantitativa, descritiva, transversal. Teve como objetivo caracterizar idosos com alteraç̃es cognitivas, usuários de Unidades de Saúde da Família (USF), que residem em diferentes contextos de vulnerabilidade social. A população estudada foi composta por pessoas com idade a partir de 60 anos, cadastrados em USFs do município de São Carlos. A coleta de dados consistiu em entrevista estruturada, utilizando-se de Genograma e Critério Brasil. Os resultados mostram que idosos que vivem em contexto de muito baixa, baixa ou média vulnerabilidade, são em sua maioria do sexo feminino, viúvos, com 80 anos de idade e mais, inseridos na classe social B. Os que vivem em contexto de alta e muito alta vulnerabilidade são predominantemente do sexo feminino, casados, com idade de até 80 anos, inseridos na classe social C. Assim, em países como o Brasil, torna-se necessária a reavaliação das estratégias de cuidado que atendam essa faixa etária emergente.

Palavras-chave: Enfermagem. Saúde do Idoso. Programa Saúde da Família.

\begin{abstract}
Study of nature quantitative, descriptive and traverse. It had as objective characterizes elderly people with cognitive alterations, users of the Family Health Units (FHU), who live in different contexts of social vulnerability. The studied population was composed by people with age from 60 years, registered in FHUs in the municipality of São Carlos. The collection of data consisted of structured interview, using Genogram and Brazil Criterion. The results show that elderly people that live in the context of very low, low or medium vulnerability, they are mostly female, widowers, with 80 years of age and plus, inserted in the social class $B$. Those who live in context of high and very high vulnerability are predominantly female, married, with age of up to 80 years, inserted in the social class C. So, in countries as Brazil, It is necessary the revaluation of the care strategies to assist this emerging age group.
\end{abstract}

Keywords: Nursing. Health of the elderly. Family Health Program.

\section{Resumen}

Estudio cuantitativo, descriptivo, transversal. Destinado a caracterizar los ancianos con cambios cognitivos, usuarios de las Unidades de Salud de la Familia (USF), que se encuentran en diferentes contextos de vulnerabilidad social. La población del estudio fue la gente mayor de 60 años de USFs registrado del municipio de São Carlos. Consistió en la recopilación de datos estructurada, utilizando el criterio Brasil y genograma. Los resultados muestran que las personas mayores que viven en el contexto de muy baja, baja o mediana vulnerabilidad, son en su mayoría mujeres, viudas, con 80 o más años de edad, clase social inserida en la clase B. Los que viven en el contexto de alta y muy alta vulnerabilidad son predominantemente de sexo femenino, casados, de 80 años, incluidos en la clase social C. Así, en países como Brasil, es necesario revisar las estrategias de atención que respondan a la nueva edad.

Palabras Clave: Enfermería. Salud del anciano. Programa de Salud Familiar.

${ }^{1}$ Enfermeira. Mestranda em Enfermagem pela Universidade Federal de São Carlos (UFSCar). São Carlos- SP-Brasil. E-mail: arieneangelini@yahoo.com.br, ${ }^{2}$ Doutora em Educação. Professora Associada do Departamento de Enfermagem da Universidade Federal de São Carlos (UFSCar).São Carlos-SP-Brasil. E-mail: sofia@ufscar.br, ${ }^{3}$ Enfermeira. Mestranda em Enfermagem pela Universidade Federal de São Carlos (UFSCar).São Carlos-SP-Brasil. E-mail: tabatta_renata@hotmail.com 


\section{INTRODUÇÃO}

0 aumento do número de idosos na população brasileira é um fato que chama atenção por suas características e consequências. Embora não seja uma exclusividade do Brasil, já que é um fenômeno universal, o aumento do tamanho da população brasileira acima dos 60 anos ocorre com algumas peculiaridades devido à velocidade com que esse processo acontece. Em países desenvolvidos, o envelhecimento vem ocorrendo de forma gradual, acompanhado de melhorias na cobertura do sistema de saúde, nas condições de habitação, saneamento básico, trabalho, alimentação e previdência social. No Brasil, ocorre rapidamente e em um contexto de desigualdades sociais, economia frágil, com precário acesso aos serviços de apoio especializado e reduzidos recursos financeiros, sem as modificações estruturais que respondam às demandas do novo grupo etário emergente. ${ }^{1}$

Os dados da Pesquisa Nacional por Amostra de Domićlios mostram que a população idosa era responsável por 7,9\% da população em 1992 e passou a constituir 10,5\% em 2007. A expectativa de vida do brasileiro em 1997 era de 69,3 anos e passou a ser de 72,7 anos em 2007 (76,5 anos para as mulheres e 69,0 anos para os homens). Atualmente a população idosa no Brasil corresponde a aproximadamente 20 milhões de pessoas. $^{2}$

Se, por um lado, o envelhecimento populacional trouxe os benefícios de uma maior longevidade, por outro, trouxe um novo perfil de morbimortalidade, caracterizado por um aumento de doenças crônico-degenerativas, como, por exemplo, as demências. ${ }^{3}$

Há a necessidade de se destacar que o envelhecimento é um fenômeno heterogêneo, ou seja, ocorre de forma diferente entre as pessoas. É sabido que há influência das condições econômicas e sociais no envelhecimento. Pessoas que vivem em situação socioeconômica precária estão mais expostas ao risco de adoecer e morrer, quadro este que se intensifica em populações vulneráveis, como os idosos. ${ }^{4}$

Somado a isto, encontram-se hoje no Brasil o despreparo e a inadequação dos serviços de saúde. Os recursos humanos, materiais e tecnológicos parecem não estar aptos a lidar com as características do envelhecimento, especialmente no que se refere ao perfil de doenças que acometem os idosos. ${ }^{5}$

$\mathrm{Na}$ literatura foram encontrados estudos que também verificaram o perfil dos idosos em diferentes contextos sociais e econômicos. Um trabalho realizado no Serviço de Neurogeriatria do Ambulatório do Hospital de Clínicas de Porto Alegre teve como sujeitos 36 idosos com doença de Alzheimer e seus respectivos cuidadores. Os resultados mostram que a maioria dos idosos era do sexo feminino $(66,7 \%)$, com idades variando de 61 a 86 anos, casados (50\%), com escolaridade em torno de quatro anos de estudo e eram "do lar" $(41,7 \%)$ antes da doença. A maior parte dos sujeitos pertencia ao nível socioeconômico $B(61,1 \%)$, e os demais ao grupo $C$ $(22,2 \%)$ e ao grupo $A(16,7 \%)$. Nenhum sujeito estava inserido em contextos de pobreza, ou seja, nos grupos D e E. ${ }^{6}$

Um estudo feito em um município do interior do Estado de São Paulo delineou o perfil de saúde de uma população idosa que morava em contexto de pobreza e era atendida por Unidades de Saúde da Família. Os resultados mostraram um predomínio de mulheres com baixa escolaridade; $24,8 \%$ referiram não receber aposentadoria ou pensão; $43,6 \%$ relataram não participar de atividades de integração social; $46,7 \%$ necessitavam de ajuda para realizar de uma a três atividades de vida diária, 74,9\% eram portadores de uma a cinco doenças crônicas não transmissíveis e 56,2\% alcançaram escores abaixo de 24 no teste cognitivo. ${ }^{4}$

Uma pesquisa realizada com 45 idosos com diagnóstico de Doença de Alzheimer e 29 cuidadores participantes da Disciplina de Geriatria da UNIFESP/EPM em São Paulo mostrou que a maioria dos idosos era feminina (72,4\%), com média etária de 79,17 anos, sendo a maior concentração de idade na faixa etária de 71 a 80 anos; $62,1 \%$ referiram uma renda mensal de até três salários mínimos; $62,1 \%$ tiveram até quatro anos de estudo e $48,3 \%$ eram viúvos. ${ }^{7}$

Com o propósito de oferecer subsídios para auxiliar na implantação de uma linha de pesquisa a idosos com alterações cognitivas no município, no âmbito da Estratégia de Saúde da Família, o presente estudo teve como objetivo caracterizar idosos com alterações cognitivas, usuários de Unidades de Saúde da Família, que residem em diferentes contextos de vulnerabilidade social em um município do interior do Estado de São Paulo.

\section{PERCURSO METOLÓGICO}

Trata-se de uma pesquisa descritiva, transversal, baseada no método quantitativo de investigação.

Foi realizada no município de São Carlos (SP), que, segundo o censo de 2000, possuía 192.998 habitantes, $11 \%$ dos quais apresentando 60 anos ou mais. ${ }^{8}$

Em dezembro de 2007 São Carlos contava com doze equipes de Saúde da Família, alocadas nas onze Unidades de Programa Saúde da Família existentes. 0 número de idosos cadastrados nas Unidades de Saúde da Família era de aproximadamente 4.700 pessoas, correspondendo a $8,7 \%$ do total de pessoas de todas as idades cadastradas.

Neste estudo foi considerado o Índice Paulista de Vulnerabilidade Social (IPVS) do setor censitário da Unidade de Saúde da Família - USF onde o idoso estava cadastrado. Este 
índice classifica os setores censitários do Estado de São Paulo de acordo com os níveis de vulnerabilidade social a que estão sujeitos os seus residentes. Essa classificação foi feita segundo as características demográficas e socioeconômicas dos moradores e engloba os setores que não possuem vulnerabilidade social até os que se encaixam na vulnerabilidade muito alta. ${ }^{9}$

Em primeiro lugar, foi realizado o levantamento das Unidades de Saúde da Família do município de São Carlos e identificado o setor censitário com o seu respectivo IPVS.

Os idosos foram identificados junto à equipe de Saúde da Família, e foram selecionados os idosos com alterações cognitivas, segundo informações do Banco de Dados do Grupo de Pesquisa Saúde e Envelhecimento.

Foram excluídas as unidades que possuíam IPVS 1, pois nesse grupo não há vulnerabilidade social. Foram incluídas as unidades inseridas em diferentes contextos de vulnerabilidade social, ou seja, IPVS 2 (Vulnerabilidade Muito Baixa), 3 (Vulnerabilidade Baixa), 4 (Vulnerabilidade Média), 5 (Vulnerabilidade Alta) e 6 (Vulnerabilidade Muito Alta). ${ }^{9}$

0 projeto foi aprovado em 6 de junho de 2008 pelo Comitê de Ética em Pesquisa da Universidade onde a pesquisa foi realizada (Parecer 253/ 2008).

Todos os cuidados éticos que regem pesquisas com seres humanos foram observados, segundo a Resolução 196/96.

A população estudada foi composta por pessoas com idade a partir de 60 anos, cadastradas e avaliadas em Unidades de Saúde da Família de regiões com diferentes índices de vulnerabilidade social do município de São Carlos. A amostra foi de 88 idosos, sendo 57 mulheres e 31 homens. Os critérios de inclusão dos participantes foram: ter 60 anos de idade ou mais; ser cadastrado em uma unidade que apresentasse o IPVS; apresentar resultado no Miniexame do Estado Mental abaixo da nota de corte, de acordo com o grau de escolaridade; não possuir comprometimentos graves de linguagem ou compreensão; assinar o Termo de Consentimento Livre e Esclarecido.

A coleta de dados teve início após a leitura e assinatura do Termo de Consentimento Livre e Esclarecido tanto pelo idoso portador de alterações cognitivas quanto pelo cuidador primário ou familiar responsável e foi realizada entre os meses de julho e dezembro de 2008.

Foram realizadas entrevistas individuais e domiciliares previamente agendadas, nos domicílios dos 88 idosos. A coleta de dados consistiu em uma entrevista estruturada, utilizando-se os instrumentos denominados Genograma e Critério Brasil.

Para a caracterização dos sujeitos, os dados foram coletados através da construção do genograma de cada família. Para essa construção, o idoso e seus familiares que estavam presentes no momento da coleta de dados foram convidados a retratar a estrutura e o histórico da família. Assim, foram levantados dados como idade, gênero, estado civil, escolaridade, renda, morbidade referida, entre outros. Foram incluídas pelo menos três gerações, sendo que as anotações foram feitas seguindo a ordem cronológica, ou seja, do mais velho para o mais novo, e registradas da esquerda para a direita no diagrama, em cada uma das gerações. Foram usados símbolos padronizados para eventos importantes como nascimento, morte, casamento e separação. No diagrama, as famílias que viviam no mesmo lar foram agrupadas. ${ }^{10}$

Para a determinação da classe social do idoso, foi utilizado o Critério Brasil. Trata-se do critério-padrão de classificação econômica da população brasileira. A padronização do sistema em todo o território brasileiro permite ao mercado comparar resultados de pesquisas realizadas em diferentes locais, empresas e períodos. Esse instrumento discrimina grandes grupos de acordo com sua capacidade de consumo de produtos e serviços acessíveis a uma parte significativa da população; classifica os domicílios, assumindo, como pressuposto, que a classe é uma característica familiar e utiliza informações objetivas e precisas de fácil coleta e operacionalização. É realizada a soma dos pontos de acordo com o grau de escolaridade do chefe da família e a posse dos itens listados nesse instrumento. A partir dessa pontuação, 0 entrevistador sabe a qual classe (A1, A2, B1, B2, C1, C2, D, E) pertence a família entrevistada.

\section{RESULTADOS}

Foram realizadas entrevistas individuais e domiciliárias com 88 idosos em diferentes contextos de vulnerabilidade social. Destes, $49 \%(n=43)$ pertencem a regiões de baixa e média vulnerabilidade social e $51 \%(n=45)$, a regiões de alta e muito alta vulnerabilidade social (Gráfico 1).

Gráfico 1 - Distribuição dos idosos por regiões de vulnerabilidade social. São Carlos, 2009.

$\square$ BAIXA E MÉDIA VULNERABILIDADE SOCIAL

- ALTA E MUITO ALTA VULNERABILIDADE SOCIAL

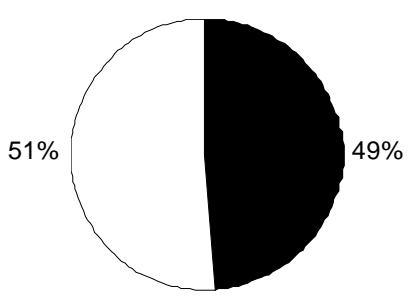

A Tabela 1 traz as características predominantes dos idosos com alterações cognitivas em diferentes contextos de vulnerabilidade social de maneira resumida 
Tabela 1 - Características predominantes dos idosos com alterações cognitivas em diferentes contextos de vulnerabilidade social. São Carlos, 2009.

\begin{tabular}{|c|c|c|}
\hline $\begin{array}{l}\text { CARACTERISTICAS } \\
\text { PREDOMINANTES }\end{array}$ & $\begin{array}{l}\text { MUITO BAIXA, BAIXA E } \\
\text { MÉDIA } \\
\text { VULNERABILIDADE } \\
\text { SOCIAL }\end{array}$ & $\begin{array}{l}\text { ALTA E MUITO ALTA } \\
\text { VULNERABILIDADE SOCIAL } \\
\text { (CONTEXTO DE POBREZA) }\end{array}$ \\
\hline Número de sujeitos & $43(49 \%)$ & $45(51 \%)$ \\
\hline Sexo & Feminino $(53 \%)$ & Feminino $(77 \%)$ \\
\hline Idade & 80 anos e mais ( $44 \%)$ & 60 aos 75 anos $(77 \%)$ \\
\hline Classe social & Classe B $(42 \%)$ & Classe C $(53 \%)$ \\
\hline Estado civil & Viủvo $(43 \%)$ & Casados $(60 \%)$ \\
\hline Escolaridade & $\begin{array}{l}\text { Ensino fundamental } \\
\text { incompleto }(44 \%)\end{array}$ & Analfabetos $(51 \%)$ \\
\hline Renda & $\begin{array}{l}\text { Meio a um salário mínimo } \\
(67 \%)\end{array}$ & Meio a um salário minimo $(69 \%)$ \\
\hline Renda proveniente de... & Aposentadoria ou pensão & Aposentadoria, pensão ou emprego \\
\hline Doença ou condição referida & $\begin{array}{l}\text { Hipertensâo arterial }(65 \%) \mathrm{e} \\
\text { diabetes }(28 \%)\end{array}$ & $\begin{array}{l}\text { Hipertensão arterial }(73 \%) \text { e } \\
\text { diabetes }(18 \%)\end{array}$ \\
\hline Convênio de saúde particular & $\operatorname{Sim}(28 \%)$ & $\operatorname{Sim}(9 \%)$ \\
\hline Tipo de cuidador & $\begin{array}{l}\text { Filha ( } 44 \% \text { ) ou profissional } \\
(14 \%)\end{array}$ & Filha $(39 \%)$ ou cônjuge ( $33 \%)$ \\
\hline
\end{tabular}

Nas regiões de muito baixa, baixa e média vulnerabilidade social, a maioria dos idosos (53\%) é do sexo feminino. Houve predomínio da faixa etária de 80 anos e mais (44\%) e da classe social B (42\%).

Amaioria dosidosos (53\%) éviúva, seguida pelos casados ou que vivem com o companheiro ( $30 \%$ ). Somente $14 \%$ deles são solteiros e nunca se casaram. Os outros $2 \%$ são separados, desquitados ou divorciados.

Em relação à escolaridade, observa-se uma significativa porcentagem de idosos com ensino fundamental incompleto (44\%), seguidos de $30 \%$ de idosos analfabetos, $16 \%$ de idosos com alfabetização de adultos, $7 \%$ com ensino fundamental completo e $2 \%$ com ensino superior completo.

Para a análise da renda dos idosos utilizou-se o valor do salário mínimo vigente na época da coleta dos dados, que era de $\mathrm{R} \$ 415,00$. A maioria dos idosos possui de meio a um salário mínimo (67\%), sendo que $60 \%$ dos idosos entrevistados são os principais responsáveis pela renda familiar. Já a renda média da família (idoso + pessoas que vivem na casa) é de um a dois salários mínimos (51\%).

Dos idosos entrevistados, $100 \%$ relatam não trabalhar.

Em relação aos problemas de saúde referidos pelosidosos, os mais citados são hipertensão arterial $(65 \%)$ seguida pelo diabetes (28\%).

Quanto aos convênios de saúde, $28 \%$ dos idosos possuem convênio particular de saúde e o restante usa o Sistema Único de Saúde, quando necessário.

Na maior parte das famílias (33\%), os idosos moram com mais uma pessoa na casa; em $23 \%$ dos casos, os idosos moram com mais duas pessoas; $16 \%$ moram sozinhos; $12 \%$ moram com mais três pessoas; $9 \%$, com cinco pessoas ou mais; e $7 \%$ moram com mais quatro pessoas. A Figura 1 apresenta o genograma de uma idosa que morava em um lar multigeracional.

Figura 1- Estrutura familiar da idosa "Maria Luzia"

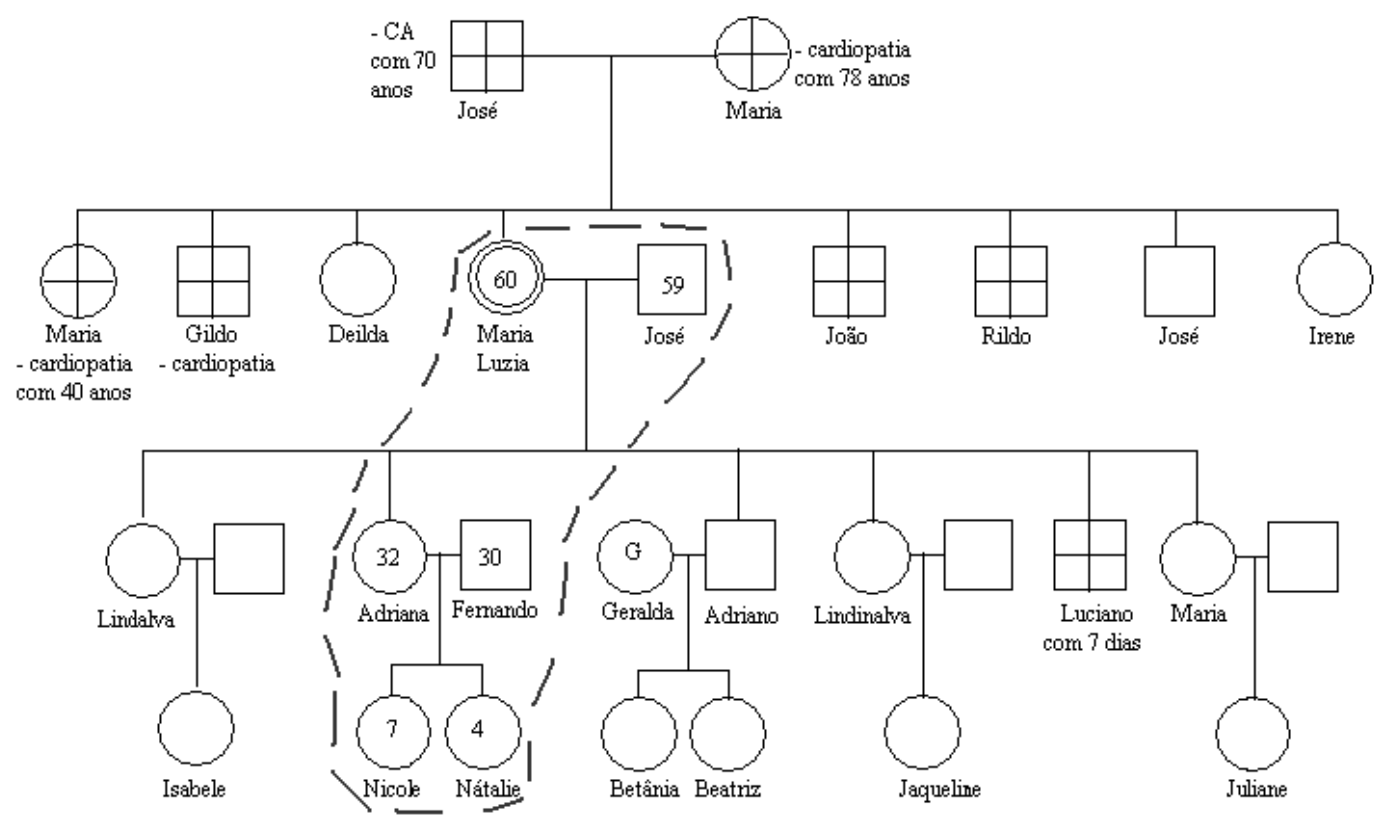


<smiles>C1CCC2CCCCC2CC1</smiles>

Mulher

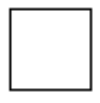

Homem

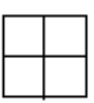

Óbito Homem

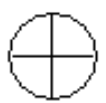

Óbito

Mulher<smiles>C1=CC(c2ccccc2)=C1</smiles>

Caso índice

Ligação normal

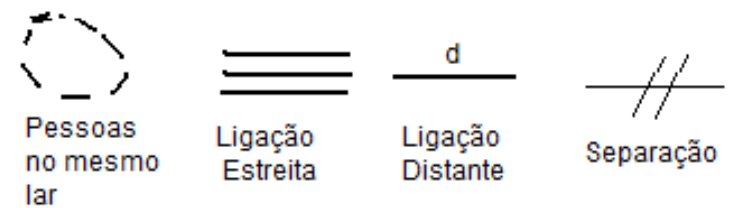

A Figura 1 apresenta a estrutura familiar da idosa "Maria Luzia", com 60 anos de idade, casada, mãe de seis filhos (um já faleceu). Morava com seu esposo "José", com sua filha "Adriana", seu genro "Fernando" e duas netas "Nátalie" e "Nicole".

$\mathrm{O}$ (a) filho(a)/enteado(a) é o parente mais citado que vive com o idoso (56\%), seguido do(a) esposo(a)/ companheiro(a) (30\%) e dos netos(as) (26\%).

Em $84 \%$ dos casos, os idosos relatam possuir um cuidador, que na maioria das vezes é a filha/enteada (44\%), seguida por um profissional em $14 \%$ dos casos. Fato semelhante ocorre quando se trata do cuidador secundário, ou seja, 33\% destes são representados pelas filhas/enteadas.

Nas regiões de alta e muito alta vulnerabilidade social, há predomínio do sexo feminino (77\%), da faixa etária dos 60 aos 75 anos (77\%) e da classe social C (53\%).

Os idosos são, na maioria dos casos, casados (60\%), seguidos pelos viúvos (29\%). A Figura 2 mostra o genograma de um idoso casado, e a Figura 3 refere-se ao genograma de um idoso viúvo.

Figura 2- Estrutura familiar do idoso "Serafim"

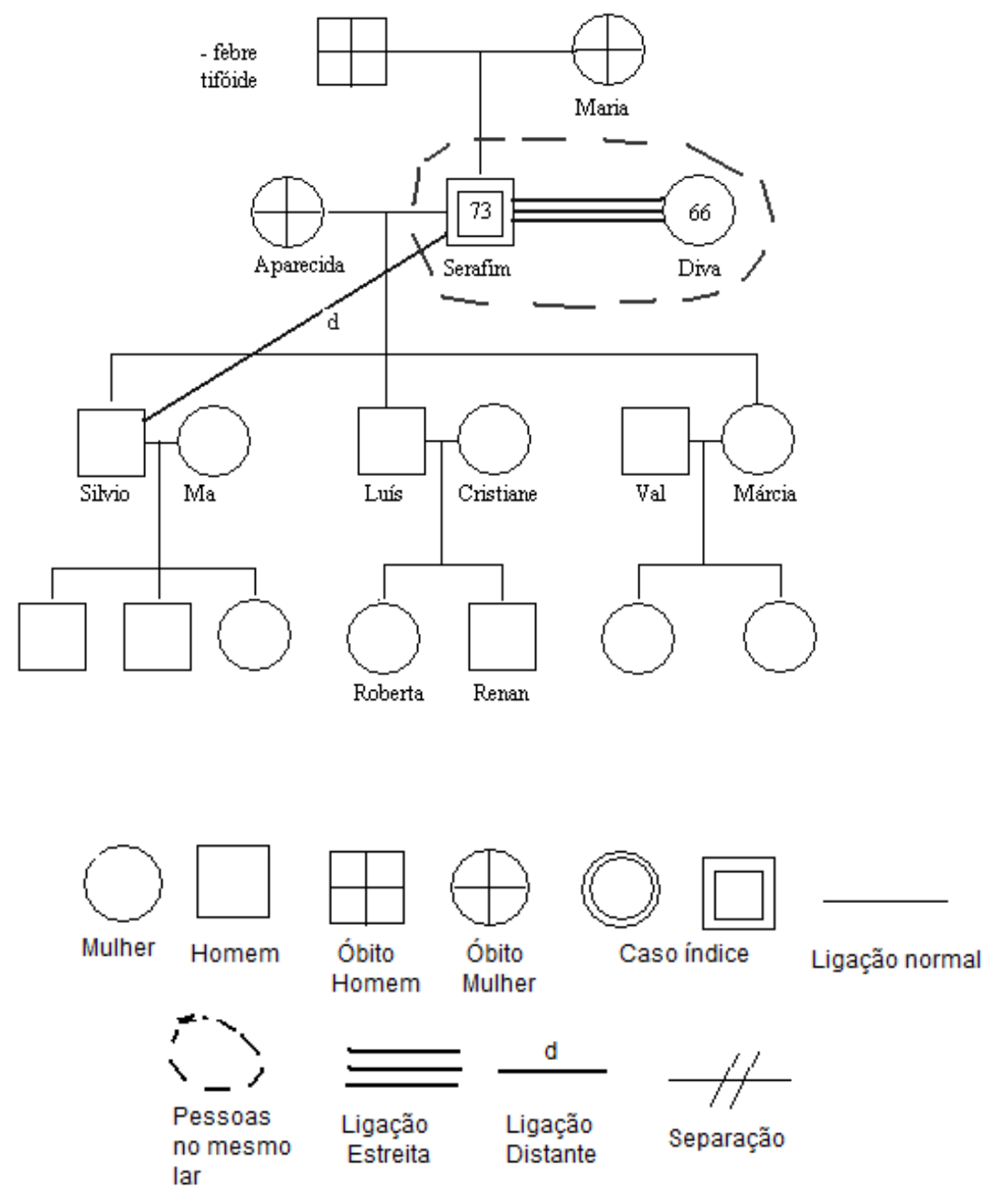


A Figura 2 apresenta a estrutura familiar do idoso "Serafim", com 73 anos de idade, casado pela segunda vez, pai de três filhos do primeiro casamento. No segundo, não teve filhos. Morava com sua esposa "Diva" (66 anos) e relatava possuir ligação estreita (linha tripla que vai de Serafim a Diva) com ela e ligação distante com seu filho mais velho "Silvio".

Figura 3- Estrutura familiar do idoso "Natanael".

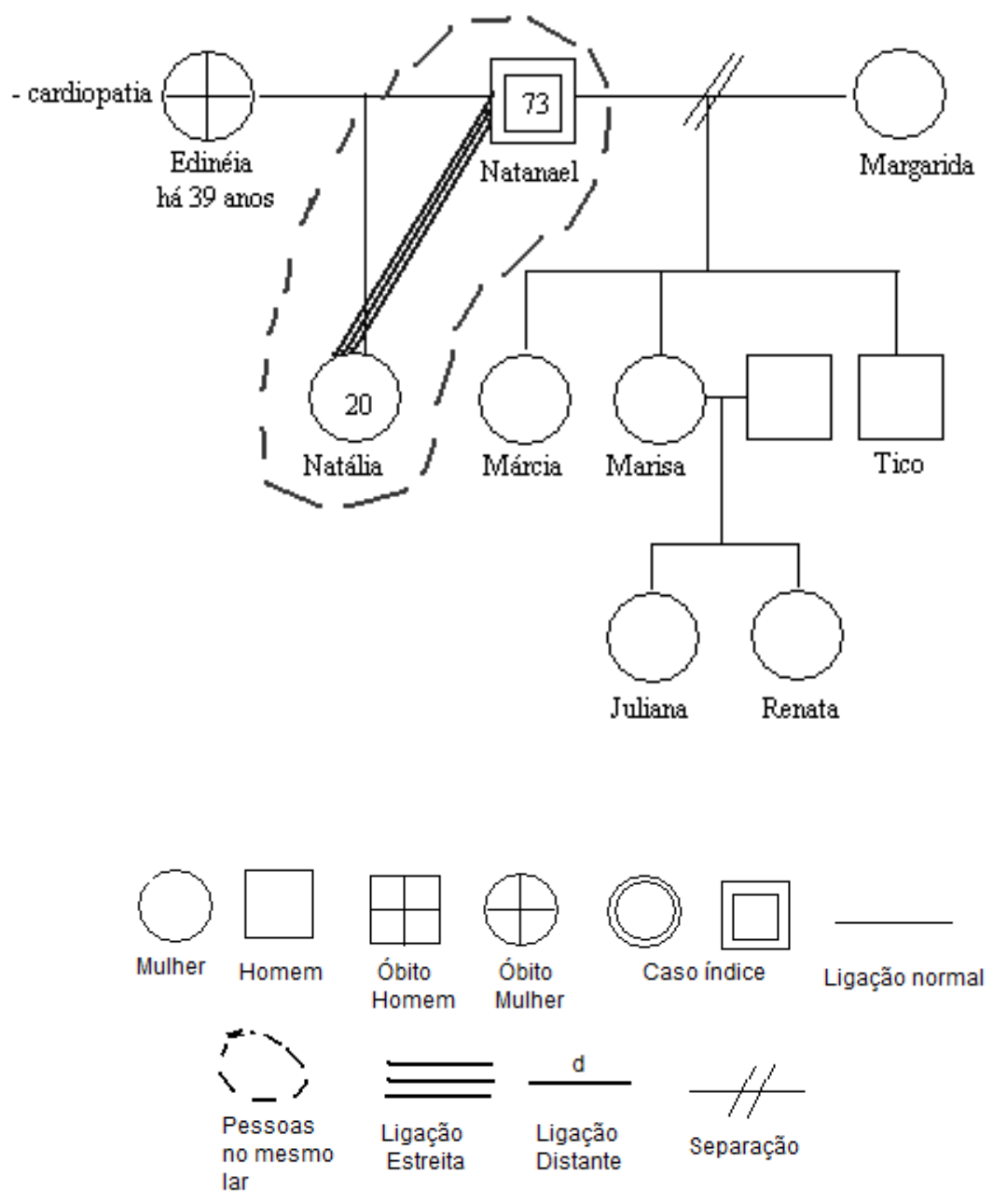

A Figura 3 apresenta a estrutura familiar do idoso "Natanael", com 73 anos de idade, atualmente viúvo, mas já se casou anteriormente com "Margarida" e se separou, pai de quatro filhos, sendo três do primeiro casamento e uma filha do segundo casamento. Morava com sua filha "Natália" e relatava possuir ligação estreita (representada na figura pela linha tripla que vai de Natanael à Natália) com ela.

Os idosos entrevistados apresentam baixa escolaridade, sendo que $51 \%$ nunca estudaram ou tiveram apenas o ensino fundamental incompleto (31\%). São os principais responsáveis pela renda familiar em $64 \%$ dos casos e possuem no máximo um salário mínimo (69\%), proveniente de aposentadoria ou pensão. A renda média dos membros da família é de, no máximo, dois salários mínimos (76\%).
A maioria é hipertensa (73\%), seguida pelos portadores de diabetes (18\%). Noventa e um porcento desses idosos não possuem convênio de saúde particular e recorrem ao Sistema Único de Saúde quando precisam de atendimento.

Sessenta porcento dos idosos vivem com seu esposo/companheiro, seguidos dos filhos $(56 \%)$ e netos (31\%). Apenas $7 \%$ deles vivem sozinhos.

Em relação aos cuidadores primários, $80 \%$ dos idosos que vivem em contextos de pobreza afirmam possuir um cuidador, sendo este as filhas/ enteadas em $39 \%$ dos casos, seguido de esposas/ companheiras em 33\% dos relatos. Quanto ao cuidador secundário, $47 \%$ são representadas pelas filhas/enteadas. 


\section{DISCUSSÃO}

0 predomínio de mulheres na população do estudo, como esperado em relação à composição demográfica de idosos (maior probabilidade de sobrevivência entre as mulheres), foi também semelhante aos resultados encontrados em outros estudos. ${ }^{4,6-7}$

Os resultados relativos ao nível de escolaridade refletem um dos aspectos de desigualdade social no país. Nesse estudo, idosos que vivem em contextos de pobreza são na maioria analfabetos ou com poucos anos de escolaridade. Achados semelhantes também foram encontrados em outras pesquisas. ${ }^{4,6-7}$

A população de idosos do estudo, tanto em contextos de alta e muito alta vulnerabilidade social quanto em contextos de muito baixa, baixa e média vulnerabilidade social, apresentou uma alta prevalência de doenças crônicas, que também foi encontrada em outros estudos. ${ }^{4-5} \mathrm{Um}$ trabalho realizado em um município do Estado de São Paulo revelou que pessoas que vivem em situação econômica precária estão mais expostas ao risco de adoecer e morrer, quadro este que se intensifica em populações mais desprotegidas ou vulneráveis, como os idosos. ${ }^{4}$

Nesse estudo, houve predominância de famílias multigeracionais. Os idosos vivem com seu cônjuge (quando ainda casados), filhos e netos. Na literatura também existem pesquisas que estão de acordo com os nossos achados. Um estudo realizado no interior do Estado de São Paulo revelou que $57 \%$ dos idosos viviam com um companheiro ou irmão, e a grande maioria (73,5\%), com os filhos. Já com os netos esta porcentagem diminui um pouco, totalizando $36,7 \% .{ }^{11}$ Estes números também foram verificados em uma outra pesquisa que observou que somente 1,9\% vivem com os bisnetos, $29,6 \%$ com os netos e 53,3\% (a maioria) com os filhos. Já os que vivem com companheiro totalizaram $36,9 \% .{ }^{4}$

A corresidência pode ser considerada uma estratégia das famílias para beneficiar tanto as gerações mais velhas como as mais jovens, e, no nosso país, isso pode significar melhora nas condições de vida. ${ }^{12}$

Várias pessoas morando na mesma residência pode ser um fator benéfico tanto para os idosos quanto para o cuidador primário, pois há maiores chances de esse idoso ser cuidado por alguém e não haver sobrecarga para uma única pessoa. Assim, a convivência intergeracional tem-se mostrado positiva à medida que membros da família assumem o papel de cuidadores secundários, minimizando situação geradora de estresse sobre a cuidadora principal. ${ }^{5}$

Em relação à renda, a maioria dos idosos não exerce atividade remunerada, sendo a renda proveniente de aposentadoria ou pensão. Segundo dados do IPEA, a aposentadoria tem-se transformado na única fonte de renda de 12 milhões de famílias brasileiras. ${ }^{13}$

Nas famílias dos idosos mais pobres, em muitos casos, o idoso é o provedor do sustento ou exerce o papel de cuidar dos netos enquanto os outros membros da família trabalham fora do espaço doméstico., ${ }^{414}$

Em relação ao cuidador, a maioria dos idosos desse estudo relatou possuir um cuidador, que na maioria das vezes é a filha e em segundo lugar o cônjuge. Geralmente, quem desempenha as tarefas de cuidar em família é uma mulher, uma vez que prestar cuidado é secularmente uma atribuição feminina, quer seja esposa, filha ou irmã. ${ }^{15}$

Estudos mostram que a filha surge como primeira opção de escolha, fato semelhante aos dados encontrados no presente estudo. ${ }^{4,6-7}$

Porém, há estudos que mostram que o cônjuge é tido como o cuidador principal, fruto da obrigação matrimonial e do dever moral estabelecido pela sociedade. ${ }^{16}$

Como as mulheres são mais longevas que os homens e, em geral, mais novas que os maridos, a cuidadora preferencial dos idosos é a esposa. Depois, vêm as descendentes de segunda geração, cuja candidata mais provável é a filha mais velha. Raramente o cuidador é outro parente ou uma pessoa jovem, e mais raramente ainda é um homem. Além de normas sociais, a elegibilidade para o papel de cuidador segue a lógica de quem está mais disponível ou mais bem preparado. ${ }^{17-18}$

\section{CONCLUSÕES}

Os resultados mostram que os idosos que vivem em contextos de muito baixa, baixa ou média vulnerabilidade social (IPVS 2, 3 e 4) ( $n=43$ ) são em sua maioria do sexo feminino, viúvos, com 80 anos de idade e mais, inseridos na classe social B de acordo com o Critério Brasil, com ensino fundamental incompleto e renda mensal de até um salário mínimo, proveniente de aposentadoria ou pensão. A maioria revelou ser hipertensa e possuir um cuidador, que geralmente é a filha.

Os idosos que vivem em contextos de alta ou muito alta vulnerabilidade social (IPVS 5 e 6$)(n=45)$ são predominantemente do sexo feminino, casados, com idade de até 80 anos, inseridos na classe social C, analfabetos e com renda mensal de até um salário mínimo, proveniente de aposentadoria, pensão ou emprego. Relataram também ser hipertensos e possuir um cuidador, que geralmente é a filha. 0 cônjuge também foi bastante citado como cuidador.

Em países como o Brasil, torna-se necessária a reavaliação das estratégias de cuidado, políticas públicas e sociais no âmbito da família e da sociedade. 0 aumento da proporção de pessoas idosas faz com que as demandas específicas deste segmento adquiram dimensões maiores. Estas políticas públicas precisam estar adequadas ao perfil da população alvo, ou seja, é necessário que haja o direcionamento dos programas para atender à essa faixa etária emergente, levando-se em consideração a diversidade da mesma. 


\section{REFERÊNCIAS}

1. Pereira RJ, Cotta RMM, Franceschini SCC, et al Contribuição dos domínios físico, social, psicológico e ambiental para a qualidade de vida global de idosos. Rev Psiquiatr 2006 jan/abr; 28(1): 27-38.

2. Instituto Brasileiro de Geografia e Estatística - IBGE. Pesquisa Nacional por Amostra de Domicílios - 2007 [citado 5 maio 2008]. Disponível em: http://www.ibge.gov.br/home/estatistica/populacao/trabalhoerendimento/ pnad2007/default.shtm

3.Alves AM. Os idosos, as redes de relações sociais e as relações familiares. In: Neri AL, organizadora. Idosos no Brasil: vivências, desafios e expectativas na terceira idade. São Paulo (SP): Ed Fundação Perseu Abramo; 2007. p.125-39.

4.Feliciano AB, Moraes AS, Freitas ICM. 0 perfil do idoso de baixa renda no Município de São Carlos, São Paulo, Brasil: um estudo epidemiológico. Cad Saude Publica 2004 nov/dez;20(6): 1575-85.

5.Gonçalves LHT, Alvarez AM, Sena ELS, Santana LWS, Vicente FR. Perfil da família cuidadora de idoso doente/fragilizado do contexto sociocultural de Florianópolis, SC. Texto\& Contexto Enferm 2006 out/ dez; 15(4): 570-77.

6 Luzardo AR, Gorini MIPC, Silva APS. Características de idosos com doença de Alzheimer e seus cuidadores: uma série de casos em um serviço de neurogeriatria. Texto\& Contexto Enferm 2006 out/dez; 15(4): 587-94.

7.Lemos ND, Gazzola JM, Ramos LR. Cuidando do paciente com Alzheimer: 0 impacto da doença no cuidador. Saude Soc 2006 set/ dez; 15(3): 170-79.

8.Instituto Brasileiro de Geografia e Estatística - IBGE . Tabela-População residente, população residente de 10 anos ou mais de idade, total, alfabetizada e taxa de alfabetização, segundo os municípios - 2000 [citado 5 maio 2008]. Disponivel em: http://www.ibge.gov.br/home/ estatistica/populacao/censo2000/universo.php?tipo $=310 /$ tabela13_1.shtm\&paginaatual $=1 \& u f=35 \& l e t r a=S$.

9.Fundação Sistema Estadual de Análise de Dados - SEADE. Índice Paulista de Vulnerabilidade Social - IPVS. Espaços e dimensões da pobreza nos municípios do Estado de São Paulo. [citado 14 fev 2008]. Disponível em: http://www.seade.gov.br/produtos/ipvs/pdf/oipvs.pdf.

10. Wright LM, Leahey M. Enfermeiras e famílias: um guia para avaliação e intervenção na família. $3^{\mathrm{a}}$ ed. São Paulo (SP): Roca; 2002.

11.Pavarini SCl, Luchesi BM, Fernandes HCL, et al. Genograma: avaliando a estrutura familiar de idosos de uma unidade de saúde da família. Rev Eletr Enferm [periódico na internet]. 2008; [citado 10 maio 2008];10(1):[aprox. 12 telas].Disponível em http://www.fen.ufg.br/revista/ v10/n1/v10n1a04.htm.

12 Camarano AA, El Ghaouri SK. Famílias com idosos: ninhos vazios? Rio de Janeiro (RJ): IPEA; 2003.
13. Areosa SVC, Areosa AL. Envelhecimento e dependência: desafios a serem enfrentados. Textos\&Contexto Enferm 2008 jan/jun;7(1):138-50.

14. Souza RFS, Skubs T, Brêtas ACP. Envelhecimento e família: uma nova perspectiva para o cuidado de enfermagem. Rev Bras Enferm 2007 maio/jun; 60(3): 263-67.

15. Neri AL, Sommerhalder C. As várias faces do cuidado e do bem-estar do cuidador. In: Neri AL, organizadora. Cuidar de idosos no contexto da família: questões psicológicas e sociais. São Paulo (SP): Alínea; 2002. p.963.

16. Karsch UMS. Idosos dependentes: familias e cuidadores. Cad Saude Publica 2003 jan; 19(3): 861-66.

17. Penning MG. Receipt of assistanse by elderly people: hierarchical selection and task specifity. Gerontologist 1991 Apr; 30(2): 220-27.

18. Braz E, Ciosak SL. 0 tornar-se cuidadora na senescência. Esc Anna Nery Rev Enferm 2009 abr/jun; 13(2): 372-77. 